\title{
Degenerate sterile neutrino dark matter in the cores of galaxies
}

\author{
F. Munyaneza ${ }^{1, \star}$ and P. L. Biermann ${ }^{1,2,3}$ \\ 1 Max-Planck-Institut für Radioastronomie, Auf dem Hügel 69, 53121 Bonn, Germany \\ e-mail: munyanez@mpifr-bonn.mpg.de \\ 2 Department of Physics and Astrononomy, University of Bonn, Germany \\ 3 Department of Physics and Astronomy, University of Alabama, Tuscaloosa, AL, USA
}

Received 29 May 2006 / Accepted 25 August 2006

ABSTRACT

\begin{abstract}
Aims. We study the distribution of fermionic dark matter at the center of galaxies using NFW, Moore and isothermal density profiles and show that dark matter becomes degenerate for particle masses of a few $\mathrm{keV}$ and for distances less than a few parsec from the center of our galaxy.

Methods. A compact degenerate core forms after galaxy merging and boosts the growth of supermassive black holes at the center of galaxies.

Results. To explain the galactic center black hole of mass of $\sim 3.5 \times 10^{6} M_{\odot}$ and a supermassive black hole of $\sim 3 \times 10^{9} M_{\odot}$ at a redshift of 6.41 in SDSS quasars, we require a degenerate core of mass between $3 \times 10^{3} M_{\odot}$ and $3.5 \times 10^{6} M_{\odot}$. This constrains the mass of the dark matter particle between $0.6 \mathrm{keV}$ and $82 \mathrm{keV}$. The lower limit on the dark matter mass is improved to $7 \mathrm{keV}$ if exact solutions of Poisson's equation are used in the isothermal power law case. We argue that the constrained particle could be the long sought dark matter of the Universe that is interpreted here as a sterile neutrino.
\end{abstract}

Key words. black hole physics - Galaxy: nucleus - cosmology: dark matter - galaxies: quasars: general

\section{Introduction}

Precision observations of the cosmic microwave background and of large scale structure confirm the picture in which $96 \%$ of the matter density of the Universe is made of dark energy and dark matter (DM), which could be revealed only by their gravitational interaction (Spergel et al. 2003). The nature of these two forms of matter is still unknown. Many candidates have been proposed for DM. These include Cold Dark Matter (CDM) particles of masses heavier than $1 \mathrm{GeV} / \mathrm{c}^{2}$ (Bertone et al. 2005) and Warm Dark Matter (WDM) particles such as sterile neutrinos (Dodelson \& Widrow 1994). Recently, there has been a renewed interest in sterile neutrinos as candidates for DM as they could be a natural extension of the minimal standard model (MSM) of electroweak interactions (Weinberg 1967; Glashow 1961). One of these sterile neutrinos could be the DM, while the other two could help explain baryogenesis (Asaka \& Shaposhnikov 2005; Shaposhnikov 2006).

Numerical cosmological $N$-body simulations suggest DM density profiles which follow $\rho \sim r^{-\gamma}$ law, with $\gamma \approx 3$ in the outer parts of the halos and $1 \lesssim \gamma \lesssim 2$ inside a few kpc (Navarro et al. 1997; Moore et al. 1998, 1999; Klypin et al. 2002 and Power et al. 2003). Although the Milky Way is well studied in the inner 3-10 kpc region, little is known about the DM distribution on smaller scales, i.e. $r \lesssim 0.1 \mathrm{pc}$ where the gravitational potential of baryons dominates over DM. In addition, there is a mounting evidence that a black hole of mass $M \sim 3.5 \times 10^{6} M_{\odot}$ dominates the mass distribution in the inner one parsec of the Galaxy (Schödel et al. 2003; Ghez et al. 2005). Thus the investigation of the distribution of DM

\footnotetext{
* Humboldt Fellow.
}

around the black hole in the Galactic center is important as it could give some hints on the nature of the DM particle (Gnedin $\&$ Primack 2005; Bertone \& Merritt 2005). Recently, we have established that the inner DM density profile scales as $\rho \sim r^{-3 / 2}$ under the assumption that the black hole feeds from degenerate fermionic DM (Munyaneza \& Biermann 2005). It was then shown that a DM particle of order of $m_{s} \sim 10 \mathrm{keV}$ could explain the growth of supermassive black holes of $10^{6}$ to $10^{9} M_{\odot}$ from stellar seed black holes. It is interesting to note that a sterile neutrino mass in overlapping mass range, with a small mixing angle with the active neutrinos, has also been suggested by Kusenko (2004) to explain the high velocities of pulsars at birth in supernovae. Moreover, a DM particle mass in the $\mathrm{keV}$ range can be constrained from X-ray background studies (Drees \& Wright 2000; Abazajian et al. 2001; Dolgov \& Hansen 2002). Here, it is worth mentioning that an upper limit of a sterile neutrino mass of $6.3 \mathrm{keV}$ from X-ray background seems to be in conflict with the lower limit of $14 \mathrm{keV}$ from SDSS Lyman-alpha forest (Abazajian \& Koushiappas 2006). However, the non-thermal phase distribution of the DM particles has the potential to modify the Lyman alpha limits, while leaving the $\mathrm{X}$-ray limits intact, so eliminating the contradiction with this additional degree of freedom. Depending on the specific model for the production of the DM particles, their initial phase space distribution is possibly largely sub-thermal. Recently, Biermann \& Kusenko (2006) established that the decay of a such sterile neutrino could speed up the formation of molecular hydrogen and boost the early star formation and reionisation in agreement with the WMAP 3-year results (Spergel et al. 2006).

The purpose of this Letter is to study the constraints on the DM particle mass in the central region of the galaxy. Given that there is still uncertainty about the DM profile around the 
central black hole, we will assume standard Navarro-FrenkWhite (NFW), Moore or isothermal gas sphere profiles and investigate what happens if the DM particles become degenerate and form a degenerate dark matter star also called a fermion ball (Munyaneza \& Viollier 2002; Munyaneza \& Biermann 2005). We then get the lower limits on the DM particle mass by assuming that the mass of the fermion ball cannot be more than the mass of $3.5 \times 10^{6} M_{\odot}$ for the Galactic center black hole. An upper limit to the DM mass will be established from the condition that the mass of the fermion ball should be greater than about $3 \times 10^{3} M_{\odot}$. The choice of this mass comes from the growth mechanism arguments to form supermassive black holes of mass of $10^{9} M_{\odot}$ in SDSS quasars (Munyaneza \& Biermann 2005). Moreover, a mass of $3 \times 10^{3} M_{\odot}$ is the upper limit of masses for which there is no black hole in galaxies such as M33 (Gebhardt et al. 2001; Barth et al. 2005). Thus the Galactic center provides us with a fertile testing ground for DM theories and cosmological structure evolution.

\section{Degenerate cores}

We study the following density profiles obtained through galaxy mergers

$\rho(r)=\rho_{0} \frac{1}{\left(\frac{r}{r_{0}}\right)^{s}} \frac{1}{\left(1+\frac{r}{r_{0}}\right)^{3-s}}$,

with $s<3$ and $\rho_{0}$ is the characteristic density at a size of $r_{0}=3 \mathrm{kpc}$. The value of $\rho_{0}$ will be found from the constraint that the DM mass enclosed at $r=3 \mathrm{kpc}$ is about $10^{10} M_{\odot}$ for the Milky Way galaxy (Klypin et al. 2002). The index $s$ characterizes the density power law slope and we will use the value of $s=1$ for NFW profile (Navarro et al. 1997), $s=1.5$ for Moore et al. $(1998,1999)$ profile and $s=2$ for the isothermal gas sphere case. The full distribution function corresponding to the the density of Eq. (1) can be found in Hansen et al. (2005). Of course, this distribution function does not allow for the Fermi Dirac nature of the DM particles. The mass enclosed within a radius $r$ and for small $r \lesssim r_{0}=3 \mathrm{kpc}$ is given by

$M(r)=\int_{0}^{r} 4 \pi r^{2} \rho(r) \mathrm{d} r=\frac{4 \pi \rho_{0}}{3-s} r_{0}^{3}\left(\frac{r}{r_{0}}\right)^{3-s}$.

The rotational velocity $v_{\text {rot }}$ corresponding to the mass enclosed $M(r)$ is

$v_{\text {rot }}=\sqrt{\frac{G M(r)}{r}}=\left(\frac{4 \pi G \rho_{0}}{3-s}\right)^{1 / 2} r_{0}\left(\frac{r}{r_{0}}\right)^{1-s / 2}$.

Here, we will consider only DM contribution to $v_{\text {rot }}$. The numerical simulation results of inner density power law density distributions suggest that for a large number of particles, their kinetic energy is strongly reduced and the more so, the deeper they are near the center. The central particle populations are essentially cooled in the core in the violent relaxation of the merger, with a local temperature $T \sim r^{2-s}$. The degeneracy condition can be written as

$\left(\frac{g_{f}}{6 \pi^{2}}\right)^{1 / 3} \frac{m_{s} v}{n^{1 / 3}}=\hbar$

Here, $m_{s}$ is the DM particle mass to be constrained, $n=\rho / m_{s}$ is the DM particle number density, $\hbar$ is the Planck's constant and $g_{f}$ is the spin degree of freedom, i.e. $g_{f}=2$ for Majorana and $g_{f}=4$ for Dirac's particles. The constraint (4) implies a degenerate Fermi Dirac distribution which of course does not have a long power law high energy tail. The Fermi Dirac distribution function $f_{\mathrm{FD}}$ is given by

$f_{\mathrm{FD}}(E)=K \frac{1}{\exp \left(\frac{E-\mu}{k T}\right)+1}, K=N\left(\int_{-\infty}^{0} \mathrm{~d} E \frac{1}{\exp \left(\frac{E-\mu}{k T}\right)+1}\right)^{-1}$

where $E$ is the DM total energy, $\mu$ the chemical potential, $T$ the temperature and $K$ is the normalisation constant and $N$ the total number of DM particles. For high energies, i.e. $E-\mu \gg k T$, the Fermi-Dirac distribution function becomes Maxwellian, i.e. $f_{\mathrm{DE}} \sim \mathrm{e}^{-(E-\mu) / k T}$ whereas direct numerical simulations favour a velocity distribution with a long power law high energy tail (Hansen \& Stadel 2006; Hansen et al. 2005) with the following profile

$f(v)=\left[1-(1-q)\left(\frac{v}{\kappa_{1} \sigma}\right)^{2}\right]^{\frac{q}{1-q}}$

where $q$ and $\kappa_{1}$ are free parameters. The latter distribution becomes Maxwellian for $q=1$. We also note that for $v \rightarrow 0$, the distribution function given by Eq. (6) becomes indistinguishable from a degenerate Fermi Dirac distribution at absolute zero Temperature. To reproduce the characteristic features of the distribution given by Eq. (6) in the Fermi Dirac distribution, we introduce a multi-temperature distribution function as follows

$f(E)=K \sum_{1}^{\infty} \frac{1}{\exp \left(\frac{E-\mu_{n}}{k T_{n}}\right)+1}$,

where $T_{n}=2^{n} T_{0}$ and the chemical potential $\mu_{n}$ is choosen from the condition that $\exp \left(\frac{\mu}{k 2^{n} T_{0}}\right)=\frac{1}{p^{n}}$. In result we get

$\mu_{n}=-k T_{0} n 2^{n} \ln p$,

where $n$ is the power index and $p^{n}$ shows by how much the distribution function has been lowered. In the limit of $n \rightarrow \infty$, the sum from Eq. (7) can be converted into the following integral

$f(E)=K \int_{T 0}^{\infty} \frac{1}{\exp \left(\frac{E-\mu}{k T}\right)+1} \frac{\mathrm{d} T}{T}$.

Thus, the chemical potential and the temperature parameters allow us to achieve a high energy power law tail in the Fermi Dirac distribution. For realistic velocity distributions obtained from large scale simulations such as given by Eq. (6), the dominant contribution to the number of particles is still the basic temperature given by $\kappa_{1} \sigma$, demonstrating that a Fermi Dirac distribution is a viable first approximation. The modification to the degeneracy criterion of Eq. (4) is small. In addition, the density powerlaw slope $s$ in NFW, Moore and isothermal distributions can be related to our parameter $p$ so that the number of particles at the temperature $T_{0}$ dominates by far over all other partial sums.

The characteristic Fermi velocity is denoted by $v$ and will be taken here as the rotational velocity, $v_{\text {rot }}$. Using Eqs. (1) and (3), we find that the degeneracy condition (4) becomes fulfilled for particle masses of a few $\mathrm{keV}$ and for distances less than a few parsec. Here, we point out that the physics of degenerate fermion balls that obey Eq. (4) was studied in a series of papers (Bilić et al. 1999; Tsiklauri \& Viollier 1998; Munyaneza et al. 1998, 1999; Munyaneza \& Viollier 2002).

Introducing the velocity dispersion $\sigma^{2}=\frac{G M_{0}}{2 r_{0}}$, where $M_{0}=$ $\frac{4 \pi r_{0}^{3} \rho_{0}}{3-s}$ is the total mass of DM contained within a size of 
$r_{0}=3 \mathrm{kpc}$. As both the velocity $v$ and the number density $n$ depend on the radial coordinate, the degeneracy condition (4) allows to get a constraint on the DM particle mass $m_{s}$ and the radius $r$. Let us denote by $R_{\mathrm{DM}}$ the radius at which DM becomes degenerate

$R_{\mathrm{DM}}=\left(\frac{6 \pi^{2}}{g_{f}}\right)^{\frac{2}{6-s}}\left(\frac{3-s}{4 \pi}\right)^{\frac{2}{6-s}} 2^{\frac{-1}{6-s}} l_{\mathrm{pl}}\left(\frac{c}{\sigma}\right)^{\frac{2}{6-s}}\left(\frac{l_{\mathrm{pl}}}{r_{0}}\right)^{\frac{s-2}{6-s}}\left(\frac{m_{\mathrm{pl}}}{m_{s}}\right)^{\frac{8}{6-s}}$

Using the last expression for the radius, we can calculate the mass $M_{\mathrm{DM}}$ of DM enclosed within the above radius $R_{\mathrm{DM}}$ to be

$M_{\mathrm{DM}}=\left(\frac{6 \pi^{2}}{g_{f}}\right)^{\frac{6-2 s}{6-s}}\left(\frac{3-s}{4 \pi}\right)^{\frac{6-2 s}{6-s}} 2^{\frac{3}{6-s}} m_{\mathrm{pl}}\left(\frac{\sigma}{c}\right)^{\frac{6}{6-s}}\left(\frac{l_{\mathrm{pl}}}{r_{0}}\right)^{\frac{3(2-s)}{6-s}}\left(\frac{m_{\mathrm{pl}}}{m_{s}}\right)^{\frac{8(3-s)}{6-s}}$

where $m_{\mathrm{pl}}=(\hbar c / G)^{1 / 2}$ and $l_{\mathrm{pl}}=\left(\hbar G / c^{3}\right)^{1 / 2}$ are the Planck's mass and length, respectively. We wish to emphasize that this is just the mass derived from the original power law density distribution, and so does not include any additional DM accretion to the DM degenerate configuration. We then rewrite the Eqs. (10), (11) for each value of the steepness index $s$. Thus, for NFW profile, i.e. $s=1$ we get

$M_{\mathrm{DM}}=5.24 m_{\mathrm{pl}}\left(\frac{\sigma}{c}\right)^{6 / 5}\left(\frac{l_{\mathrm{pl}}}{r_{0}}\right)^{3 / 5}\left(\frac{m_{\mathrm{pl}}}{m_{s}}\right)^{16 / 5}$,

$R_{\mathrm{DM}}=1.62 l_{\mathrm{pl}}\left(\frac{c}{\sigma}\right)^{2 / 5}\left(\frac{l_{\mathrm{pl}}}{r_{0}}\right)^{-1 / 5}\left(\frac{m_{\mathrm{pl}}}{m_{s}}\right)^{8 / 5}$,

for the total mass and the size of the degenerate core, respectively. For Moore profile, i.e. $s=1.5$, the total mass $M_{\mathrm{DM}}$ and the size $R_{\mathrm{DM}}$ of the degenerate core are given by

$M_{\mathrm{DM}}=3.68 m_{\mathrm{pl}}\left(\frac{\sigma}{c}\right)^{4 / 3}\left(\frac{l_{\mathrm{pl}}}{r_{0}}\right)^{1 / 3}\left(\frac{m_{\mathrm{pl}}}{m_{s}}\right)^{8 / 3}$,

$R_{\mathrm{DM}}=1.50 l_{\mathrm{pl}}\left(\frac{c}{\sigma}\right)^{4 / 9}\left(\frac{l_{\mathrm{pl}}}{r_{0}}\right)^{-1 / 3}\left(\frac{m_{\mathrm{pl}}}{m_{s}}\right)^{16 / 9}$,

and finally for the isothermal gas sphere, i.e. for $s=2$, the mass $M_{\mathrm{DM}}$ and the size $R_{\mathrm{DM}}$ scale as

$M_{\mathrm{DM}}=2.58 m_{\mathrm{pl}}\left(\frac{\sigma}{c}\right)^{3 / 2}\left(\frac{m_{\mathrm{pl}}}{m_{s}}\right)^{2}, R_{\mathrm{DM}}=1.29 l_{\mathrm{pl}}\left(\frac{c}{\sigma}\right)^{1 / 2}\left(\frac{m_{\mathrm{pl}}}{m_{s}}\right)^{2}$.

In order to investigate the behaviour of the degenerate DM mass $M_{\mathrm{DM}}$ and its size $R_{\mathrm{DM}}$ as functions of the DM mass $m_{s}$, we fix the total DM mass $M_{0}=10^{10} M_{\odot}$ at a distance of $r_{0}=$ $3 \mathrm{kpc}$ and this gives a velocity dispersion of $\sigma=84.65 \mathrm{~km} \mathrm{~s}^{-1}$ (Ferrarese \& Merritt 2000) for the three density profiles. Here, only DM contribution to the total mass is taken into account.

We then assume that the total mass of the degenerate core should be between $3 \times 10^{3} M_{\odot}$ and $3.5 \times 10^{6} M_{\odot}$. There are two arguments for the choice of this lower limit on the mass of fermion ball. First, this mass is needed to boost the growth of stellar seed black holes to $\sim 10^{6} M_{\odot}$ which then grow to $\sim 10^{9} M_{\odot}$ supermassive black hole in SDSS quasars by baryonic accretion (Munyaneza \& Biermann 2005). Secondly, this is the threshold mass, below which no black hole will be formed as in the case of M33 (Gebhardt et al. 2001; Barth et al. 2005).

For NFW profile, the mass of the DM particle should be in the range of $0.6 \mathrm{keV} \lesssim m_{s} \lesssim 6 \mathrm{keV}$. A range of $1 \mathrm{keV} \lesssim$ $m_{s} \lesssim 14 \mathrm{keV}$ is found for Moore profile and finally we obtain

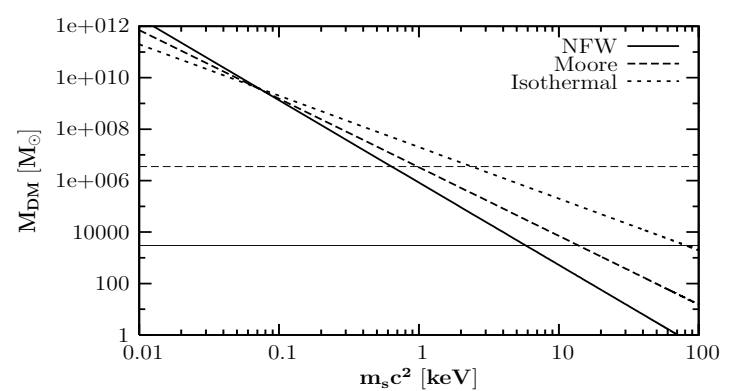

Fig. 1. The total mass $M_{\mathrm{DM}}$ of the fermion ball as a function of the fermion mass $m_{s}$. The total mass $M_{\mathrm{DM}}$ scales with the the DM particle mass $m_{s}$ as $m_{s}^{-16 / 5}, m_{s}^{-8 / 3}$ and as $m_{s}^{-2}$ for NFW, Moore and isothermal power law, respectively. Two horizontal lines at $M_{\mathrm{DM}}=3.5 \times 10^{6} M_{\odot}$ and $M_{\mathrm{DM}}=3 \times 10^{3} M_{\odot}$ have been drawn to get the lower and upper limits on the mass of the DM particle.

$2 \mathrm{keV} \lesssim m_{s} \lesssim 82 \mathrm{keV}$ for the isothermal gas sphere. These limits on the DM particle mass are illustrated in Fig. 1 where we plot the total mass of the degenerate core as a function of the DM mass $m_{s}$. Using these lower limits for the DM particle, we predict a density of $(1-100) M_{\odot} \mathrm{pc}^{-3}$ in the case of NFW and Moore profiles at the size of a few parsec, which corresponds to the values obtained in Klypin et al. (2002). In the case of the isothermal gas sphere, we predict a much higher density of a few $10^{5} M_{\odot} \mathrm{pc}^{-3}$ at a size of about $1 \mathrm{pc}$.

Here, we want to point out that the total mass of DM core in the isothermal gas sphere case is increased by a factor of nine if we use the full numerical solutions of Poisson's equation for a self gravitating system of fermions (Munyaneza \& Biermann 2005). Thus, in order to get a degenerate core of $3.5 \times$ $10^{6} M_{\odot}$ with a velocity dispersion of $\sigma=84.65 \mathrm{~km} \mathrm{~s}^{-1}$, we get a fermion mass of $m_{s} \approx 7 \mathrm{keV}$ instead of lower limit of $m_{s} \sim 2 \mathrm{keV}$. Using a fully developed distribution function such as the one given in Eq. (9) would result in further changes of the obtained limits. However, we do not expect any major modifications since the number of particles in the tail is much smaller compared to the main body of the phase space distribution.

\section{Conclusion and discussion}

We have determined the constraints on the DM mass under the assumption that the NFW, Moore or isothermal density profiles become degenerate near the center. Assuming that the mass of the degenerate fermion ball is between about $3 \times 10^{3} M_{\odot}$ and $3.5 \times 10^{6} M_{\odot}$, we have found that the mass of the DM particle should have a lower limit of about $7 \mathrm{keV}$ in the isothermal power law case. The above limit on the DM mass is obtained from a simple model which does not take into account baryonic matter and a proper model for DM with anisotropic distribution in phase space (Evans \& An 2006; Hansen \& Stadel 2006) and baryonic matter would modify these limits. The obtained mass range is in full agreement with the Tremaine-Gunn lower bound of about $0.5 \mathrm{keV}$ on the mass of any fermionic DM when applied to dwarf spheroidal galaxies (Tremaine \& Gunn 1979). The constraints obtained on DM particle mass overlap with the upper limit on sterile neutrino masses of about $8 \mathrm{keV}$ to obey the X-ray emission constraints from the Virgo cluster observations (Abazajian 2005). In addition, a sterile neutrino mass in the mass range of 2-20 keVs was derived to explain the high velocities up to $1000 \mathrm{~km} \mathrm{~s}^{-1}$ experienced by pulsars at birth in supernovae (Kusenko 2004; Barkovitch et al. 2004; Fuller et al. 2003). Moreover, the obtained constraints agree well with the lower 
bound of $m_{s} \approx 2 \mathrm{keV}$ on the mass of sterile neutrinos obtained from the analysis of the Lyman- $\alpha$ forest data (Hansen et al. 2002; Viel et al. 2005; Abazajian 2005). Recently, Biermann \& Kusenko (2006) have shown that the decay of such a sterile neutrino could help initiate star formation in the early Universe and the detection of such an X-ray line would confirm whether such a sterile neutrino exists or not and this could be done by observing the X-ray decay line using XMM and Chandra satellites (Boyarski et al. 2006; Riemer-Srensen et al. 2006).

To summarize, we find that NFW, Moore and isothermal density profiles become degenerate for fermion DM particle of $\mathrm{keV}$ masses at a size of the order parsec. Assuming that supermassive black holes grow from degenerate fermion cores of masses of a few $10^{3} M_{\odot}$ to $\sim 10^{6} M_{\odot}$ at galactic centers, we have found that the sterile neutrino mass should be in the range from $0.6 \mathrm{keV}$ to about $82 \mathrm{keV}$, with an improved lower limit of about $7 \mathrm{keV}$ in the isothermal power law density profile. As this range of sterile neutrino masses overlaps with other results on sterile neutrino masses discussed in this Letter, this leaves the sterile neutrino to be an excellent candidate for DM. The formation and growth details of degenerate fermion balls is beyond the scope of this Letter and will be discussed in another paper.

Acknowledgements. We are grateful to Alex Kusenko and Raoul Viollier for useful discussions at SNAC06 in Crans-Montana. We also thank Mikhail Shaposhnikov for discussions at the Marcell Grossman meeting (MG11) in Berlin. FM thanks the organisers of SNAC06 for the hospitality in Crans-Montana and he acknowledges the financial support from the Alexander von Humboldt foundation. The work of PLB is supported by the Pierre Auger grant $05 \mathrm{CU}$ 5PD1/2 via DESY/BMF.

\section{References}

Asaka, T., \& Shaposhnikov, M. 2005, Phys. Rev. Lett. B, 620, 17

Abazajian, K. 2005 [arXiv: astro-ph/0511630]

Abazajian, K. 2006, Phys. Rev. D, 73, 063513

Abazajian, K., Fuller, G. M., \& Patel, M. 2001, Phys. Rev. D, 64, 023501

Abazajian, K., \& Koushiappas, S. M. 2006 [arXiv:astro-ph/0605271]

Barkovitch, M., D’Olivo, J. C., \& Montemayor, R. 2004, Phys. Rev. D, 70, 043005
Barth, A., Greene, J. E., \& Ho, L. C. 2005, ApJ, 619, L151

Bertone, G., Hooper, D., \& Silk, J. 2005, Phys. Rep., 405, 279

Bertone, G., \& Merritt, D. 2005, Phys. Rev. D, 72, 103502

Biermann, P. L., \& Kusenko, A. 2006, Phys. Rev. Lett., 96, 091301

Boyarsky, A., Neronov, A., Ruchayskiy, O., Shaposhnikov, M., \& Tkachev, I. 2006 [arXiv: astro-ph/0603660]

Bilić, N., Munyaneza, F., \& Viollier, R. D. 1999, Phys. Rev. D., 59, 024003 itional ref. 15/06/06

Dodelson, S., \& Widrow, L. M. 1994, Phys. Rev. Lett., 72, 17

Dolgov, A. D., \& Hansen, S. H. 2002, Astr. Part. Phys., 16, 339

Drees, M., \& Wright, D. 2000, [arXiv: hep-ph/0006274]

Ferrarese, L., \& Merritt, D. 2000, ApJ, 539, L9

Fuller, G. M., Kusenko, A., Mocioiu, I., \& Pascoli, S. 2003, Phys. Rev. D, 68, 1003002

Gebhardt, K., Richstone, D., Kormendy, J., et al. 2000, AJ, 119, 1157

Gebhardt, K., Kormendy, J., Pinkney, J., et al. 2001, ApJ, 122, 2469

Ghez, A., Salim, S., Hornstein, S. D., et al. 2005, ApJ, 620, 744

Glashow, S. L. 1961, Nucl. Phys., 22, 579

Gnedin, O. Y., \& Primack, J. R. 2004, Phys. Rev. Lett., 93, 061302

Hansen, S. H., \& Stadel, J. 2006, JCAP, 0605, 014

Hansen, S. H., Lesgourgues, J., Pastor, S., \& Silk, J. 2002, MNRAS, 333, 544

Hansen, S. H., Egli, D., Hollenstein, L., \& Salzmann, C. 2005, New Astr., 10, 379

Evans, N. W., \& An, J. H. 2006 [arXiv:astro-ph/0511687]

Klypin, A., Zhao, H., \& Somerville, R. 2002, ApJ, 573, 597

Kogut, A., Spergel, D. N., Barnes, C., et al. 2003, ApJS, 148, 161

Kusenko, A. 2004, Int. Journ. Mod. Phys. D, 13, 2065

Moore, B., Governato, F., Quinn, T., et al. 1998, ApJ, 499, L5

Moore, B., Ghigna, S., Governato, F., et al. 1999, ApJ, 524, L19

Munyaneza, F., \& Biermann, P. L. 2005, Astr. Astrophys., 436, 805

Munyaneza, F., \& Viollier, R. D. 2002, ApJ, 564, 274

Munyaneza, F., Tsiklauri, D., \& Viollier, R. D. 1998, ApJ, 509, L105

Munyaneza, F., Tsiklauri, D., \& Viollier, R. D. 1999, ApJ, 526, 744

Navarro, J. F., Frenk, C. S., \& White, S. D. M. 1997, ApJ, 490, 493

Power, C., et al. 2003, MNRAS, 338, 14

Riemer-Srensen, S., Hansen, S. H., \& Pedersen, K. 2006, ApJ, 644, L33

Schödel, R., Ott, T., Genzel, R., et al. 2003, ApJ, 596, 1015

Shaposhnikov, M. 2006, [arXiv: hep-ph/060547]

Spergel, D. N., Verde, L., Peiris, H. V., et al. 2003, ApJS, 148, 175

Spergel, D. N., Bean R., Doré, O., et al. 2006 [arXiv: astro-ph/0603449]

Tremaine, S., \& Gunn, J. E. 1979, Phys. Rev. Lett., 42, 407

Tsiklauri, D., \& Viollier, R. D. 1998, ApJ, 500, 591

Viel, M., Lesgourgues, J., Haehnelt, M. G., Matarrese, S., \& Riotto, A. 2005, Phys. Rev. D71, 063534

Viollier, R. D. 1994, Prog. Part. Nucl. Phys, 32, 51

Weinberg, S. 1967, Phys. Rev. Lett., 19, 1264 